\title{
Seed Sources for Florida Homegrown Vegetables ${ }^{1}$
}

\author{
Ed Thralls, Sydney Park Brown, and Ed Paulson²
}

The right vegetable varieties can make a big difference in the success of a home vegetable garden. Although a huge selection of seeds and transplants are available through garden centers, seed catalogs, and the internet, choosing what to buy can be confusing.

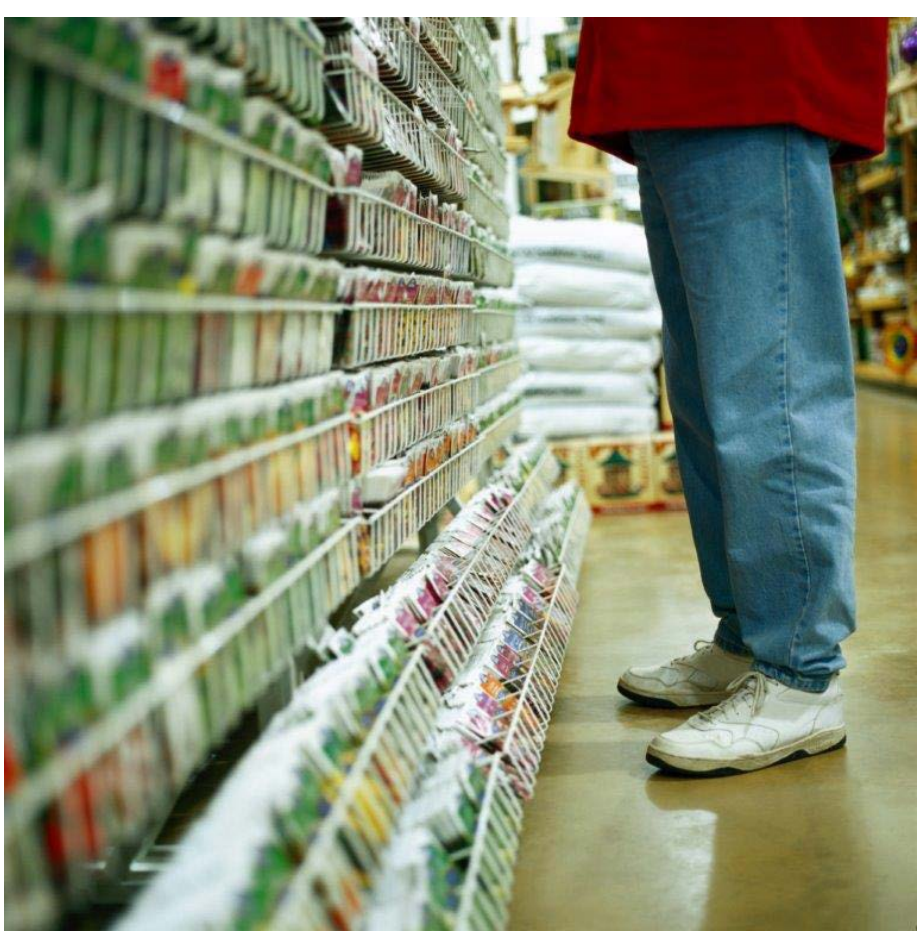

Seed sources include online catalogs and local garden centers Credits: Photodisc
It's best to start with the vegetables you and your family like to eat. Then, refer to the varieties listed in the Florida Vegetable Gardening Guide, which is available at http://edis. ifas.ufl.edu/vh021. Unfortunately, at times, these recommended varieties can be difficult to find from mail-order seed catalogs, internet suppliers, and local garden supply stores. Since some of these sources also charge shipping and handling fees, it can become costly to buy varieties from different sources.

To overcome this, consider buying from the seed company (catalog or internet) that offers the most of your desired varieties. Then, buy missing varieties locally from seed stores or substitute them. Anything marked "All-America Selections" (AAS) is usually a good substitute because these varieties were tested in nationwide trials for superior adaptability and vigor. Look for seeds that are disease and pest resistant or tolerant, which is usually noted on the packet label or indicated by a series of letters, such as V, F, N, and T. (For more information about "All-America Selections," please visit http://www.all-americaselections.org/about/ index.cfm.)

Many new varieties are introduced each year, but a new selection's performance can only be determined by actually growing it. Be sure to keep notes on what vegetable varieties were planted and how they performed. Do not save seeds from hybrid varieties because they will not grow true to type and may produce poor-quality vegetables.

1. This document is ENH1225, one of a series of the Environmental Horticulture Department, UF/IFAS Extension. Original publication date November 2013. Visit the EDIS website at http://edis.ifas.ufl.edu.

2. Ed Thralls, Extension agent III, UF/IFAS Extension, Orange County, Orlando, FL; Sydney Park Brown, associate professor and Extension specialist - Consumer Horticulture, Department of Environmental Horticulture; Ed Paulson, master gardener volunteer, UF/IFAS Extension Orange County, Orlando, FL. UF/IFAS Extension Gainesville, FL 32611 
Some of the best varieties for Florida gardens are listed in Table 1. The numbers listed beside them correspond to the seed suppliers that currently sell them in Table 2 . The exclusion of other varieties or seed companies in no way indicates that they are undesirable.

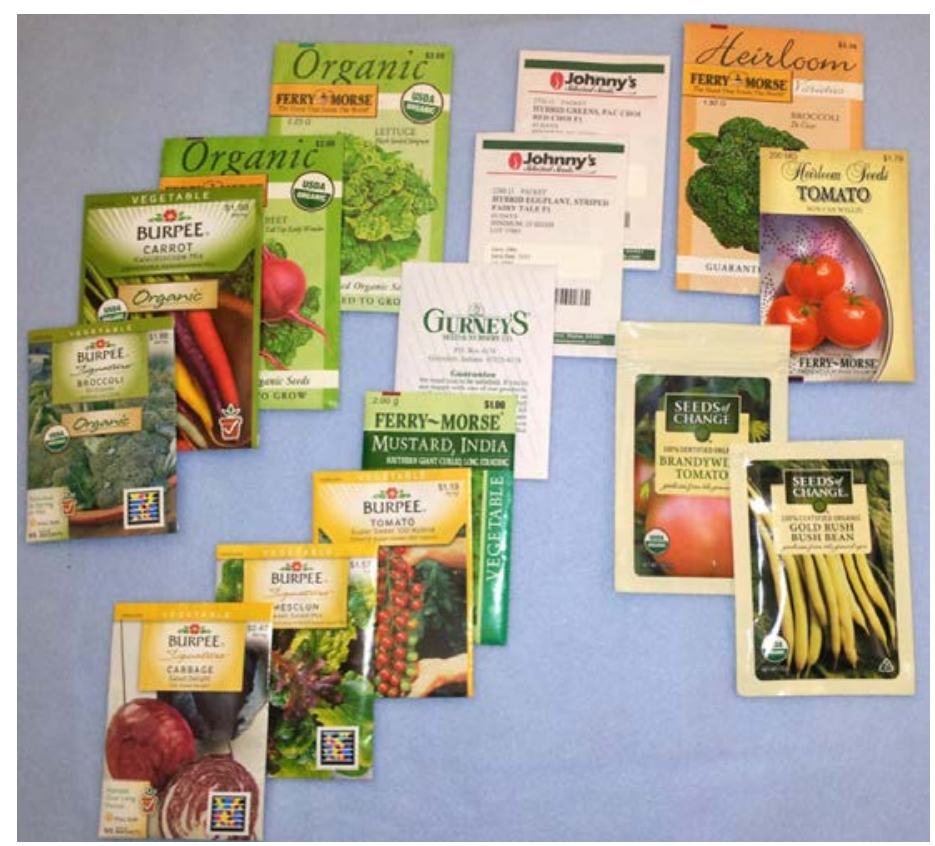

Some vegetable varieties perform better in Florida gardens than others.

Credits: Ed Thralls

\section{References}

"All-America Selections." 1311 Butterfield Road, Suite 310, Downers Grove, Il 60515-5625. http://www.allamericaselections.org/about/

Bonina, Jennifer and Cantliffe, Daniel J. 2009. Seed Production and Seed Sources of Organic Vegetables. HS981. Horticultural Sciences Department. UF/IFAS Extension:

Gainesville, FL. http://edis.ifas.ufl.edu/HS227

Park Brown, S., Stephens, J.M., Treadwell, D., Webb, S., Gevens, A., Dunn, R.A., Kidder, G., Short, D., and Simone, G.W. 2012. Florida Vegetable Gardening Guide. SP103.

Horticultural Sciences Department. UF/IFAS Extension: Gainesville, FL. http://edis.ifas.ufl.edu/VH021 
Table 1. Vegetable varieties and sources

\begin{tabular}{|c|c|c|c|}
\hline Vegetable/Variety & Source (*See Table 2) & Vegetable/Variety & Source (*See Table 2 ) \\
\hline $\begin{array}{l}\text { Beans } \\
\text { Snap } \\
\text { Bush Blue Lake } \\
\text { Contender } \\
\text { Roma II } \\
\text { Provider } \\
\text { Cherokee Wax }\end{array}$ & $\begin{array}{l}\text { 1-2-3-7-8-11-13-14-15-16-19-23 } \\
4-7-11-13-15-23 \\
4-6-7-11-12-13-1416-18-20 \\
4-10-11-15-16-19-20 \\
6\end{array}$ & $\begin{array}{l}\text { Cantaloupes and } \\
\text { Honeydews } \\
\text { Athena } \\
\text { Ambrosia } \\
\text { Galia (green flesh) }\end{array}$ & $\begin{array}{l}2-4-7-11-12-13-20 \\
11-13-14-22-23 \\
12\end{array}$ \\
\hline $\begin{array}{l}\text { Bush Type: } \\
\text { Shell } \\
\text { Horticultural } \\
\text { Pinto } \\
\text { Red Kidney } \\
\text { Black Bean } \\
\text { Navy }\end{array}$ & $\begin{array}{l}4 \\
13 \\
10 \\
10 \\
5\end{array}$ & $\begin{array}{l}\text { Carrots } \\
\text { Imperator } \\
\text { Nantes } \\
\text { Danvers } \\
\text { Chantenay }\end{array}$ & $\begin{array}{l}4-6-13-15-16-23 \\
2-3-6-7-11-14-18-19 \\
1-3-6-7-13-14-15-16 \\
1-3-12-13-16-18-19\end{array}$ \\
\hline $\begin{array}{l}\text { Pole Type: } \\
\text { McCaslan } \\
\text { Kentucky Wonder } \\
\text { Blue Lake }\end{array}$ & $\begin{array}{l}4-15-16 \\
2-3-4-6-7-11-13-14-15-16-18-23 \\
3-6-7-11-12-13-14-15-16-19\end{array}$ & $\begin{array}{l}\text { Cauliflower } \\
\text { Snowball } \\
\text { Snow Crown } \\
\text { Brocoverde }\end{array}$ & $\begin{array}{l}1-2-3-6-13-18 \\
7-10-11-14-16-22-23 \\
3-12\end{array}$ \\
\hline $\begin{array}{l}\text { Lima: } \\
\text { Fordhook } 242 \\
\text { Henderson } \\
\text { Jackson } \\
\text { Wonder } \\
\text { Dixie (Speckled) Butterpea } \\
\text { Early Thorogreen }\end{array}$ & $\begin{array}{l}2-6-7-10-14-16-23 \\
1-4-15-16-23 \\
1-16-20 \\
4 \\
1-14-15-16-23 \\
5-16-23\end{array}$ & $\begin{array}{l}\text { Celery } \\
\text { Utah Strains }\end{array}$ & $3-6-13-15-20$ \\
\hline $\begin{array}{l}\text { Beets } \\
\text { Tall Top } \\
\text { Early Wonder } \\
\text { Detroit Dark Red } \\
\text { Cylindra } \\
\text { Red Ace } \\
\text { Yellow Detroit }\end{array}$ & $\begin{array}{l}\text { 6-19 } \\
1-3-6-10-13-15-16-20 \\
1-2-3-4-6-7-13-14-15-18-23 \\
1-2-3-10-11-12-13-15-23 \\
10-11-13-14-16-20-23 \\
6-18\end{array}$ & $\begin{array}{l}\text { Collards } \\
\text { Georgia } \\
\text { Georgia Southern } \\
\text { Top Bunch } \\
\text { Vates }\end{array}$ & $\begin{array}{l}3-6-14 \\
6-13 \\
4 \\
4-13-16\end{array}$ \\
\hline $\begin{array}{l}\text { Cabbage } \\
\text { Rio Verde } \\
\text { Flat Dutch } \\
\text { Round Dutch } \\
\text { Wakefield types } \\
\text { Copenhagen Market } \\
\text { Savoy } \\
\text { Red Acre }\end{array}$ & $\begin{array}{l}4 \\
1-2-4-12-13-16 \\
4-6 \\
1-3 \\
24 \\
4-9-11-23 \\
16-19\end{array}$ & $\begin{array}{l}\text { Corn, sweet } \\
\text { Silver Queen (white) } \\
\text { How Sweet It Is (white) } \\
\text { Sweet Ice (white) } \\
\text { Sweet Riser (yellow) } \\
\text { Early Sunglow (yellow) }\end{array}$ & $\begin{array}{l}2-3-4-6-7-14-16 \\
14-16 \\
8 \\
24 \\
3-6-14\end{array}$ \\
\hline $\begin{array}{l}\text { Cucumbers } \\
\text { Slicers: } \\
\text { Sweet Success } \\
\text { Poinsett } \\
\text { Ashley } \\
\text { MarketMore } 76 \\
\text { Straight Eight } \\
\text { Space Master } \\
\text { Pickers: } \\
\text { Eureka } \\
\text { Boston Pickling }\end{array}$ & $\begin{array}{l}3-7-9-14-16-23 \\
4-6 \\
4-11-12-13-23 \\
1-3-4-6-10-11-13-14-16-19-23 \\
1-2-3-4-6-7-9-11-13-14-16-18 \\
3-6-9-16 \\
7-9-22 \\
1-4-6-13-16\end{array}$ & $\begin{array}{l}\text { Kale } \\
\text { Vates Dwarf Blue Curled } \\
\text { Tuscan (Lacinato) } \\
\text { Winterbor } \\
\text { Redbor }\end{array}$ & $\begin{array}{l}1-4-6-13-16 \\
3-10-12-14 \\
4-10-12-14-20 \\
14-18-20\end{array}$ \\
\hline $\begin{array}{l}\text { Eggplant } \\
\text { Black Beauty } \\
\text { Dusky } \\
\text { Long } \\
\text { Ichiban } \\
\text { Cloud Nine (white) }\end{array}$ & $\begin{array}{l}1-2-3-4-6-9-13-14-16-18 \\
15 \\
6-13 \\
18 \\
15\end{array}$ & $\begin{array}{l}\text { Kohlrabi } \\
\text { Early White Vienna } \\
\text { Purple Vienna }\end{array}$ & $\begin{array}{l}1-3-6-7-16 \\
1-3-4-13-16-23\end{array}$ \\
\hline
\end{tabular}




\begin{tabular}{|c|c|c|c|}
\hline $\begin{array}{l}\text { Endive } \\
\text { Green Curled Ruffec }\end{array}$ & $6-11$ & $\begin{array}{l}\text { Lettuce } \\
\text { Crisphead: } \\
\text { Great Lakes } \\
\text { Butterhead: } \\
\text { Ermosa } \\
\text { Bibb } \\
\text { Tom Thumb } \\
\text { Buttercrunch } \\
\text { Loose Leaf: } \\
\text { Simpson types } \\
\text { Salad Bowl } \\
\text { Red Sails } \\
\text { New Red Fire } \\
\text { Oak Leaf: } \\
\text { Salad Bowl } \\
\text { Royal Oak } \\
\text { Romaine: } \\
\text { Paris Island Cos } \\
\text { Outrageous }\end{array}$ & $\begin{array}{l}6-13 \\
10 \\
3-6 \\
1-3-6-19-20 \\
2-3-6-7-10-11-13-14-16-18-20-23 \\
3-4-6-9-16-20-23 \\
3-10-12-14 \\
3-6-7-10-13-20-23 \\
17-18 \\
3-6-10-16 \\
3-16-18 \\
3-10-11-13-18 \\
10-12-19-1\end{array}$ \\
\hline $\begin{array}{l}\text { Escarole } \\
\text { Batavian Broadleaf }\end{array}$ & 5 & $\begin{array}{l}\text { Mustard } \\
\text { Southern Giant Curled } \\
\text { Florida Broad Leaf } \\
\text { Tender Green } \\
\text { Giant Red } \\
\text { Green Wave } \\
\text { Mizuna }\end{array}$ & $\begin{array}{l}3-4-6-11-14-16-18 \\
3-4-6 \\
3-4-16-18 \\
3-10-14-18 \\
8-17-18 \\
3-18\end{array}$ \\
\hline $\begin{array}{l}\text { Okra } \\
\text { Clemson Spineless } \\
\text { Emerald } \\
\text { Annie Oakley II } \\
\text { Cajun Delight }\end{array}$ & $\begin{array}{l}1-3-4-6-7-9-10-13-14-16-18 \\
1-6 \\
7-9-14 \\
11-23\end{array}$ & $\begin{array}{l}\text { Peppers } \\
\text { Bell: } \\
\text { California Wonder } \\
\text { Red Knight } \\
\text { Big Bertha } \\
\text { Other Sweet: } \\
\text { Sweet Banana } \\
\text { Giant Marconi } \\
\text { Mariachi } \\
\text { Cubanelle } \\
\text { Jalapeno: } \\
\text { Early Jalapeno } \\
\text { Jalapeno M } \\
\text { Specialty Hot: } \\
\text { Cherry Bomb } \\
\text { Hungarian Hot Wax } \\
\text { Big Chili II } \\
\text { Numex } \\
\text { Ancho } \\
\text { Thai } \\
\text { Anaheim Chile } \\
\text { Long Cayenne } \\
\text { Habanero } \\
\text { Caribbean Red Habanero }\end{array}$ & $\begin{array}{l}1-2-3-6-13-14-16-18-19-20 \\
9 \\
9-14-16 \\
2-4-7-9-16 \\
3-9 \\
3-13-14 \\
3-4-6-7-12 \\
6-9-10-14-16-20 \\
3-4-6-14 \\
3-9-16 \\
1-12-13-14-16-19 \\
9-16 \\
9-19 \\
9-10-12-13-14 \\
1-3-14 \\
2-3-4-9-13 \\
3-6-9-13 \\
1-3-4-6-9-10-13-20 \\
12\end{array}$ \\
\hline $\begin{array}{l}\text { Onions } \\
\text { Bulbing: } \\
\text { Granex (yellow) } \\
\text { Bunching (green): } \\
\text { Evergreen Bunching } \\
\text { White Lisbon Bunching } \\
\text { Leeks: } \\
\text { American Flag } \\
\text { Multiplier: } \\
\text { Shallots }\end{array}$ & $\begin{array}{l}3-4-6-9-12-14 \\
3-6-7-10-11-16 \\
3-6 \\
5-6 \\
2-3-9-12-14-19-23\end{array}$ & $\begin{array}{l}\text { Potatoes, Irish } \\
\text { Red Pontiac } \\
\text { Yukon Gold } \\
\text { Gold Rush }\end{array}$ & $\begin{array}{l}2-14-16 \\
2-3-14-16 \\
5\end{array}$ \\
\hline
\end{tabular}




\begin{tabular}{|c|c|c|c|}
\hline $\begin{array}{l}\text { Peas } \\
\text { English or Snow: } \\
\text { Wando } \\
\text { Green Arrow } \\
\text { Sugar Snap } \\
\text { Oregon Sugarpod II }\end{array}$ & $\begin{array}{l}1-2-3-6-16 \\
13-14-19-20 \\
1-2-3-7-10-13-14-16-18-19-20 \\
1-2-3-4-6-7-18-19-20\end{array}$ & $\begin{array}{l}\text { Potatoes, Sweet } \\
\text { Centennial } \\
\text { Beauregard } \\
\text { Vardaman }\end{array}$ & $\begin{array}{l}3-13-14 \\
3-6-8-14 \\
3-14-21\end{array}$ \\
\hline $\begin{array}{l}\text { Peas } \\
\text { Southern: } \\
\text { California Blackeye No. } \\
\text { Pinkeye Purple Hull } \\
\text { Texas Cream }\end{array}$ & $\begin{array}{l}2-4-6-16 \\
6-16 \\
15-22\end{array}$ & $\begin{array}{l}\text { Pumpkin } \\
\text { Big Max } \\
\text { Connecticut Field } \\
\text { Prizewinner } \\
\text { Jack Be Little } \\
\text { Jack O Lantern }\end{array}$ & $\begin{array}{l}1-6-7-13-16 \\
1-7-13-16 \\
3-9-15 \\
4-6-9-13-20 \\
3-6-16\end{array}$ \\
\hline $\begin{array}{l}\text { Radish } \\
\text { Cherry Belle } \\
\text { White Icicle } \\
\text { Sparkler } \\
\text { Champion } \\
\text { Daikon }\end{array}$ & $\begin{array}{l}3-6-7-13-16-20-23 \\
3-6-11-12-16 \\
6-11-14 \\
4-6-7-11-13-16-23 \\
3-4-6-11-16-23\end{array}$ & $\begin{array}{l}\text { Strawberry } \\
\text { Chandler } \\
\text { Oso Grande } \\
\text { Sweet Charlie } \\
\text { Selva } \\
\text { Camarosa } \\
\text { Festival }\end{array}$ & $\begin{array}{l}\text { Local garden centers may offer these } \\
\text { varieties (as plants) in early Fall. }\end{array}$ \\
\hline $\begin{array}{l}\text { Spinach } \\
\text { Melody } 3 \\
\text { Bloomsdale Longstanding } \\
\text { Tyee } \\
\text { Space }\end{array}$ & $\begin{array}{l}3 \\
1-3-4-6-7-11-13-16-20-23 \\
6-7-11-14-16-20-23 \\
11-14-20-23\end{array}$ & $\begin{array}{l}\text { Swiss Chard } \\
\text { Bright Lights } \\
\text { Bright Yellow } \\
\text { Fordhook Giant } \\
\text { Lucullus } \\
\text { Red Ruby }\end{array}$ & $\begin{array}{l}3-6-7-11-12-13-14-15-16-20-22-23 \\
15 \\
1-3-13-15-16-18-19-20-23 \\
7-13 \\
6-13-18\end{array}$ \\
\hline $\begin{array}{l}\text { Squash } \\
\text { Summer: } \\
\text { Early Prolific Straightneck } \\
\text { Summer Crookneck } \\
\text { Early White Scallop } \\
\text { Winter: } \\
\text { Spaghetti } \\
\text { Table King } \\
\text { Table Queen \& Table Ace } \\
\text { Waltham } \\
\text { Early Butternut } \\
\text { Zucchini: } \\
\text { Cocozelle } \\
\text { Spineless Beauty } \\
\text { Black Beauty } \\
\text { Calabaza }\end{array}$ & $\begin{array}{l}1-2-3-4-6-7-12-16-18 \\
3-4-6-12-13-14 \\
1-6-11-13-16 \\
1-2-3-711-12-13-16-18-20 \\
6-11 \\
1-2-3-13 \\
3-4-11-13-16-18-20-23 \\
1-3-6-11-12-13-14-23 \\
1-6 \\
4-6-11-13-14-23 \\
1-2-4-6-11-13-14-16-18-20 \\
\text { Latin Markets - seeds from whole fruit }\end{array}$ & $\begin{array}{l}\text { Tomatoes } \\
\text { Large Fruit: } \\
\text { Celebrity } \\
\text { Heat Wave II } \\
\text { Better Boy } \\
\text { Beefmaster } \\
\text { BHN444 - Southern Star } \\
\text { Amelia } \\
\text { BHN640 } \\
\text { Small Fruit: } \\
\text { Sweet 100 } \\
\text { Juliet } \\
\text { Red Grape } \\
\text { Sun Gold } \\
\text { Sugar Snack } \\
\text { Sweet Baby Girl } \\
\text { Heirloom: } \\
\text { Green Zebra } \\
\text { Cherokee Purple } \\
\text { Eva Purple Ball } \\
\text { Mortgage Lifter Delicious } \\
\text { Brandywine }\end{array}$ & $\begin{array}{l}2-7-13-14-16-21 \\
3 \\
2-3-6-7-9-11-14-21-22 \\
6-9-13-14- \\
15-20 \\
22 \\
21 \\
6-9-14-21 \\
3-6-13-14-21-22 \\
1 \\
9-21-22 \\
3-15 \\
3-7-9-14 \\
1-3-12-13-20-21 \\
1-3-14-16-18-20-21-22 \\
8-15 \\
7-14-163-7-16-21 \\
1-3-4-6-7-9-11-12-13-14-16-18-20-21-23\end{array}$ \\
\hline $\begin{array}{l}\text { Turnips } \\
\text { Roots: } \\
\text { Purple Top White Globe } \\
\text { Greens: } \\
\text { Seven Top } \\
\text { Shogoin }\end{array}$ & $\begin{array}{l}1-3-4-6-7-11-13-16-23 \\
13-16 \\
16\end{array}$ & $\begin{array}{l}\text { Watermelon } \\
\text { Large: } \\
\text { Jubilee } \\
\text { Crimson Sweet } \\
\text { Charleston Grey } 133 \\
\text { Small: } \\
\text { Sugar Baby } \\
\text { Mickeylee }\end{array}$ & $\begin{array}{l}4-16 \\
1-3-4-6-7-9-11-13-16-18-19-23 \\
1-2-3-4-9 \\
1-2-3-6-7-9-12-16-19-20 \\
4\end{array}$ \\
\hline
\end{tabular}


Table 2. Seed suppliers

\begin{tabular}{|c|c|c|c|c|c|}
\hline $\begin{array}{c}\text { Source } \\
\text { Key }\end{array}$ & Company & Website & $\begin{array}{c}\text { Source } \\
\text { Key }\end{array}$ & Company & Website \\
\hline 1 & $\begin{array}{l}\text { Baker Creek Heirloom } \\
\text { Seeds }\end{array}$ & http://rareseeds.com & 13 & Mountain Valley Seed & http://www.mvseeds.com \\
\hline 2 & $\begin{array}{l}\text { Burgess Seed and } \\
\text { Plant }\end{array}$ & http://www.eburgess.com & 14 & Park Seed & http://parkseed.com \\
\hline 3 & Burpee & http://www.burpee.com & 15 & $\begin{array}{l}\text { Pinetree Garden } \\
\text { Seeds and } \\
\text { Accessories }\end{array}$ & https://www.superseeds.com \\
\hline 4 & Clifton Seed & http://www.cliftonseed.com & 16 & RH Shumway's & http://www.rhshumway.com \\
\hline 5 & EverWilde Farms & http://www.everwilde.com & 17 & Reimer Seeds & http://www.reimerseeds.com \\
\hline 6 & Ferry Morse Seed & http://www.plantationproducts.com & 18 & Richters & http://www.richters.com \\
\hline 7 & $\begin{array}{l}\text { Gurney's Seeds and } \\
\text { Nursery }\end{array}$ & http://gurneys.com & 19 & Seeds of Change & http://www.seedsofchange.com \\
\hline 8 & Harris Seed & http://www.Harrisseed.com & 20 & Territorial Seed & http://www.territorialseed.com \\
\hline 9 & $\begin{array}{l}\text { HPS Horticultural } \\
\text { Products and Services }\end{array}$ & http://www.hpsseed.com & 21 & $\begin{array}{l}\text { Tomato Growers } \\
\text { Supply }\end{array}$ & http://www.tomatogrowers.com \\
\hline 10 & $\begin{array}{l}\text { Johnny's Selected } \\
\text { Seeds }\end{array}$ & http://www.johnnyseeds.com & 22 & Totally Tomatoes & http://www.totallytomato.com \\
\hline 11 & Jung Seeds & http://www.jungseed.com & 23 & Vermont Bean Seed & http://www.vermontbean.com \\
\hline 12 & Kitchen Garden Seeds & http://www.kitchengardenseeds.com & 24 & Victory Seeds & http://www.victoryseeds.com \\
\hline
\end{tabular}

